\title{
Two Brothers with Skewed Thiopurine Metabolism in Ulcerative Colitis Treated Successfully with Allopurinol and Mercaptopurine Dose Reduction
}

\author{
Frank Hoentjen · Stephen B. Hanauer • \\ Nanne K. de Boer • David T. Rubin
}

Received: 5 September 2011 / Accepted: 22 November 2011/Published online: 7 December 2011

(C) The Author(s) 2011. This article is published with open access at Springerlink.com

\begin{abstract}
Thiopurine therapy effectively maintains remission in inflammatory bowel disease. However, many patients are unable to achieve optimum benefits from azathioprine or 6-mercaptopurine because of undesirable metabolism related to high thiopurine methyltransferase (TPMT) activity characterized by hepatic transaminitis secondary to increased 6-methylmercaptopurine (6-MMP) production and reduced levels of therapeutic 6-thioguanine nucleotide (6-TGN). Allopurinol can optimize this skewed metabolism. We discuss two brothers who were both diagnosed with ulcerative colitis (UC). Their disease remained active despite oral and topical mesalamines. Steroids followed by 6-mercaptopurine (MP) were unsuccessfully introduced for both patients and both were found to have high 6-MMP and low 6-TGN levels, despite normal TMPT enzyme activity, accompanied by transaminitis. Allopurinol was introduced in combination with MP dose reduction. For both brothers addition of allopurinol was associated with successful remission and optimized MP metabolites. These siblings with active UC illustrate that skewed thiopurine metabolism may occur despite normal TPMT enzyme activity and can lead to adverse events in the absence of disease control. We confirm previous data
\end{abstract}

F. Hoentjen $(\bowtie) \cdot$ S. B. Hanauer · D. T. Rubin

Inflammatory Bowel Disease Center, University of Chicago,

Chicago, IL, USA

e-mail: frankhoentjen@gmail.com

F. Hoentjen

Department of Gastroenterology, Radboud University Medical Center, Nijmegen, The Netherlands

N. K. de Boer

Department of Gastroenterology and Hepatology,

VU University Medical Center, Amsterdam, The Netherlands showing that addition of allopurinol can reverse this skewed metabolism, and reduce both hepatotoxicity and disease activity, but we now also introduce the concept of a family history of preferential MP metabolism as a clue to effective management for other family members.

Keywords 6-Mercaptopurine - Allopurinol · Azathioprine $\cdot$ TPMT $\cdot$ Ulcerative colitis

\section{Introduction}

Thiopurine therapy, including 6-mercaptopurine (MP) and azathioprine, is a well-established steroid-sparing maintenance strategy for inflammatory bowel disease (IBD). However, many patients discontinue this therapy because of lack of efficacy or intractable adverse events [1]. For some of these patients thiopurine metabolism is skewed; this is characterized by grossly increased levels of 6-methylmercaptopurine (6-MMP) and reduced levels of the pharmacologically active 6-thioguanine nucleotides (6-TGN) in red blood cells (RBC). This skewed 6-MMP/ 6-TGN ratio is associated with hepatotoxicity (mainly transaminitis), pancytopenia, and lack of therapeutic response [2-5].

Thiopurine $S$-methyltransferase (TPMT) is an enzyme that catalyzes the conversion of azathioprine and MP to 6-MMP. Genetic polymorphisms of TPMT have been described and homozygous and heterozygous allelic variations occur in 0.3 and $5-15 \%$ of the general population, respectively [6-9]. These genetic polymorphisms correlate with TPMT enzyme activity and accurately predict absent to low (homozygous genotype) and intermediate (heterozygous genotype) levels of TPMT enzyme activity $[10,11]$. Low TPMT enzyme activity generally results in a 
reduced 6-MMP/6-TGN ratio, because thiopurines are shunted to the therapeutic 6-TGN, leading to an increased risk of myelotoxicity [12]. Conversely, a normal "wildtype" TPMT genotype is predictive of normal to high enzyme activity and these patients typically produce larger amounts of 6-MMP, associated with a reduced likelihood of achieving clinical remission [13, 14].

One strategy used to overcome this unfavorable metabolic profile characterized by a high 6-MMP/6-TGN ratio is addition of allopurinol to adjusted low-dose thiopurine therapy. This combination therapy leads to increased formation of 6-TGN and a decrease in 6-MMP levels. Several studies have demonstrated that this strategy can optimize thiopurine metabolism in IBD patients, with steroid-free remission achieved in more patients and with reduced adverse events [15-17].

In this case report we discuss two brothers diagnosed with UC. Both had a high 6-MMP/6-TGN ratio despite normal TPMT enzyme activity and regular weight-based MP monotherapy. Addition of allopurinol combined with reduction of MP dose led to full clinical remission and resolution of liver test abnormalities. This case report emphasizes the significance of optimization of thiopurine therapy by allopurinol co-administration for IBD patients with skewed metabolism, and further demonstrates the potential familial nature of this metabolic pattern.

\section{Cases}

Patient A is a 22-year-old male who developed hematochezia at the age of 16 . Colonoscopy by his pediatrician revealed diffuse inflammation of the large intestine with more intense inflammation in the distal colon He responded promptly to corticosteroid therapy, but his remission was not durable despite therapy with oral and topical mesalamine. MP (50 mg) was initiated to enable steroid withdrawal after normal TPMT enzyme activity $(23.0 \mathrm{U} / \mathrm{ml}$ RBC, normal $>21 \mathrm{U} / \mathrm{ml}$ RBC) was demonstrated (Table 1). However, during attempts to increase the dose of MP, levels of alanine aminotransferase (ALT) reached a maximum of $114 \mathrm{U} / 1$ (normal <35), without clinical signs of adverse effects. Subsequent measurement of thiopurine metabolites revealed high levels of 6-MMP of $13,477 \mathrm{pmol} / 8 \times 10^{8} \mathrm{RBC}$, whereas 6 -TGN remained at subtherapeutic levels $\left(<235 \mathrm{pmol} / 8 \times 10^{8} \mathrm{RBC}\right)$. Reduction of the MP dose to $75 \mathrm{mg}$ resulted in normalization of liver tests and lower levels of thiopurine metabolites (6-TGN 137 and 6-MMP 3,700 pmol/8 $\times 10^{8}$ ). In the next year, the patient was not able to achieve steroid-free remission with MP and oral and topical mesalamine. Allopurinol $100 \mathrm{mg}$ was introduced to skew the MP metabolism toward higher 6-TGN levels and lower 6-MMP levels. The dose of MP was reduced to $25 \mathrm{mg}$ to prevent a disproportionate increase in 6-TGN levels that could potentially cause myelotoxicity. He responded well to this strategy over the course of the following months. He achieved full clinical remission in the absence of hepatotoxicity or myelotoxicity and was able to discontinue corticosteroids. The dose of MP was increased to $50 \mathrm{mg}$ daily to obtain optimum levels of 6-TGN, on the basis of MP metabolites. Recent measurement of thiopurine metabolites showed a 6-TGN level of $422 \mathrm{pmol} / 8 \times 10^{8} \mathrm{RBC}$ and 6-MMP was not quantifiable (lower detection level $\left.255 \mathrm{pmol} / 8 \times 10^{8} \mathrm{RBC}\right)$. On follow-up 14 months after initiation of the combination therapy, he remains in stable, steroid-free remission on allopurinol $100 \mathrm{mg}$ and MP $50 \mathrm{mg}$.

Case B is a 19-year-old male and the younger brother of the patient described in case A. In 2008, he was diagnosed with mild-to-moderate ulcerative proctitis. Histology of rectal biopsies showed moderately active colitis compatible with UC. He improved on oral and topical mesalamine. In the subsequent year his symptoms recurred, and an increased dose of oral mesalamines, mesalamine enemas, and short courses of corticosteroids resulted in temporary clinical improvement only. Endoscopically, his proctitis progressed to left-sided UC and he was admitted to our IBD service. Intravenous administration of methylprednisolone was initiated with only minimal clinical improvement by day 5 . Subsequently, he received a loading dose of infliximab $5 \mathrm{mg} / \mathrm{kg}$ in order to induce remission. He noticed improvement of symptoms and was discharged. However, he shortly had recurrence of his symptoms and, despite escalation of the infliximab to $10 \mathrm{mg} / \mathrm{kg}$ and addition of hydrocortisone enemas, was

Table 1 Characteristics of thiopurine therapy for cases A and B

\begin{tabular}{llllllll}
\hline Case & \multicolumn{2}{l}{ MP monotherapy } & & & \multicolumn{3}{l}{ MP/allopurinol } \\
\cline { 2 - 3 } \cline { 6 - 8 } & TPMT & 6-TGN & 6-MMP & ALT & & 6-TGN & 6-MMP \\
\hline A & 23.0 & 137 & 13,477 & 114 & 422 & ALT \\
B & 19.7 & 301 & 12,796 & 141 & 351 & NQ & NQ \\
\hline
\end{tabular}

MP, 6-mercaptopurine; TPMT, thiopurine methyltransferase, normal $>21 \mathrm{U} / \mathrm{ml} \mathrm{RBC}$; 6-TGN, 6-thioguanine nucleotide, $\mathrm{pmol} / 8 \times 10^{8} \mathrm{RBC}$; 6-MMP, 6-methylmercaptopurine, pmol/8 $\times 10^{8} \mathrm{RBC}$; ALT, alanine aminotransferase, normal <35 U/l; NQ, not quantifiable 
unable to achieve symptom control. Infliximab was discontinued because of the absence of clinical benefit. His TMPT was determined at $19.7 \mathrm{U} / \mathrm{ml} \mathrm{RBC}$ and MP was initiated at approximately $1.5 \mathrm{mg} / \mathrm{kg}$. Within a few weeks after initiation of this thiopurine therapy, his symptoms continued and we noticed elevation of his ALT to $141 \mathrm{U} / \mathrm{l}$. The MP metabolites were determined and showed a 6-TGN level of 301 and elevated 6-MMP levels (12,796 pmol/ $8 \times 10^{8} \mathrm{RBC}$ ). Subsequently, the dose of MP was reduced to $25 \mathrm{mg}$ and allopurinol $100 \mathrm{mg}$ per day was started. He promptly responded to allopurinol with shunting of his thiopurine metabolites toward the formation of 6-TGN (351 pmol/8 $\times 10^{8} \mathrm{RBC}$ ) and undetectable 6-MMP levels, which clinically corresponded with complete resolution of his transaminitis. He rapidly achieved stable remission with this strategy. We continue to follow him and he has currently been in stable remission for 8 months, maintained by MP $50 \mathrm{mg}$ and allopurinol $100 \mathrm{mg}$ per day.

\section{Discussion}

In this report we discuss two siblings who were diagnosed with UC at a young age. The subsequent course of their disease and the therapy required were remarkably similar. They both had steroid-dependent disease and required steroid-sparing maintenance strategy. After introduction of MP, they had a skewed 6-MMP/6-TNG ratio with transaminitis and poor disease control. Full clinical remission of UC was achieved with resolution of earlier liver test abnormalities after optimizing thiopurine therapy by addition of allopurinol to low-dose MP.

Rundles and others have demonstrated that addition of allopurinol increased the bioavailability of MP [18]. However, myelotoxicity frequently occurred with this combination therapy and, subsequently, allopurinol was mainly used for treatment of gout. Combination therapy with thiopurines and allopurinol was used again in transplantation medicine to optimize immunosuppression in renal transplant patients [19]. In the last decade, our group and other groups have reported clinical benefit from addition of allopurinol to dose-adjusted thiopurine therapy in IBD patients. Although small in size, these series have demonstrated a clinically relevant optimization of the skewed 6-MMP/6-TGN ratio [15-17]. This combination therapy is associated with steroid-free remission, and with reduction in transaminitis corticosteroid dosing and nonhepatic adverse events that occurred in IBD patients on thiopurine monotherapy [15-17].

It is unclear by which mechanism allopurinol improves thiopurine metabolism. One potential mechanism is inhibition of TPMT through 6-thioxanthine (6-TX), as demonstrated in in-vitro studies in RBC [20]. This is an attractive explanation because allopurinol facilitates the production of 6-TX when combined with low-dose thiopurines. Alternatively, oxypurinol metabolites, which antagonize TPMT, may be involved [21]. The outcomes of in-vitro studies are, unfortunately, limited, because inhibitory metabolites are washed off during the TPMT assay. Therefore, in-vivo studies are required to test these hypotheses and to seek alternative pathways in IBD patients. The results of these two cases suggest the mechanism of preferential 6-MMP shunting may have a genetically determined origin.

High TPMT enzyme activity (wild type) generally results in reduced availability of MP for 6-TGN generation, because of rapid methylation of MP to 6-MMP. These patients have difficulty achieving therapeutic 6-TGN levels and tend to develop high levels of 6-MMP with the attendant higher risk of transaminitis. We determined TPMT enzyme activity for both siblings in this case report; their TPMT enzyme activity was in the low-normal range and they still developed elevated levels of methylated metabolites of MP. TPMT genotyping was not available for the patients in our case report. However, it is unlikely that their skewed thiopurine metabolism is the result of previously described allelic TPMT variations that would lead to a homozygous or heterozygous TPMT genotype given their similar normal rather than low to absent TPMT enzyme activity and excellent phenotype-genotype correlation for this group of patients in the literature [10, 11]. Alternatively, allelic variations correlating with high TPMT enzyme activity and resulting high 6-MMP levels have been described but do not apply to our patients because they had low-normal rather than high TPMT enzyme activity [22].

The familial aspect of skewed thiopurine metabolism with a high 6-MMP/6-TGN ratio as presented in this report is rare. In previously published cases familial genetic variations led to altered TPMT enzyme activity in multiple family members, but these were only cases with reduced to absent TPMT enzyme activity [23, 24]. As far as we are aware, no hereditary pattern associated with a high 6-MMP/6-TGN ratio has yet been reported.

The finding of skewed thiopurine metabolism despite normal TPMT enzyme activity is not uncommon. In our yet unpublished cohort at the University of Chicago that included a subgroup of 26 IBD patients with a high 6-MMP/6-TGN ratio, 19 (73\%) patients had low to normal TPMT enzyme activity. In this group of patients, and the patients discussed in this case report, TPMT status has poor predictive value for therapeutic efficacy and adverse events and recent studies confirm our observations. For example, TPMT enzyme activity was not predictive of treatment outcome a in a recent prospective trial in 113 IBD patients who started thiopurine therapy [25]. Furthermore, in both 
the latter study and a recent meta analysis, TPMT status was not predictive of transaminitis [10, 25]. Thus, TPMT status has limitations as a predictor of thiopurine metabolism, clinical efficacy, and adverse events.

In conclusion, we discuss two siblings who developed transaminitis and therapeutic failure, despite low-normal TPMT enzyme activity, when exposed to normal weightbased MP for active UC. Addition of allopurinol and reduction of MP dose led to normalization of the 6-MMP/ 6-TGN ratio and liver test abnormalities, and steroid-free remission was achieved for both siblings. This is the first report of a familial skewed MP metabolism of this pattern and is indicative of underlying genetic determination of this metabolism, possibly similar to the well described TPMT polymorphism.

Open Access This article is distributed under the terms of the Creative Commons Attribution Noncommercial License which permits any noncommercial use, distribution, and reproduction in any medium, provided the original author(s) and source are credited.

\section{References}

1. Jharap B, Seinen ML, de Boer NK, et al. Thiopurine therapy in inflammatory bowel disease patients: analyses of two 8-year intercept cohorts. Inflamm Bowel Dis. 2010;16:1541-1549. doi: 10.1002/ibd.21221.

2. Dubinsky MC, Lamothe S, Yang HY, et al. Pharmacogenomics and metabolite measurement for 6-mercaptopurine therapy in inflammatory bowel disease. Gastroenterology. 2000;118:705713. doi:S0016508500286216.

3. Cuffari C, Hunt S, Bayless T. Utilisation of erythrocyte 6-thioguanine metabolite levels to optimise azathioprine therapy in patients with inflammatory bowel disease. Gut. 2001;48:642-646.

4. Osterman MT, Kundu R, Lichtenstein GR, Lewis JD. Association of 6-thioguanine nucleotide levels and inflammatory bowel disease activity: a meta-analysis. Gastroenterology. 2006;130:10471053. doi:10.1053/j.gastro.2006.01.046.

5. Gilissen LP, Derijks LJ, Verhoeven HM, et al. Pancytopenia due to high 6-methylmercaptopurine levels in a 6-mercaptopurine treated patient with Crohn's disease. Dig Liver Dis. 2007;39:182186. doi:10.1016/j.dld.2006.05.026.

6. Collie-Duguid ES, Pritchard SC, Powrie RH, et al. The frequency and distribution of thiopurine methyltransferase alleles in Caucasian and Asian populations. Pharmacogenetics. 1999;9:37-42.

7. Engen RM, Marsh S, Van Booven DJ, McLeod HL. Ethnic differences in pharmacogenetically relevant genes. Curr Drug Targ. 2006; 7:1641-1648.

8. Hon YY, Fessing MY, Pui CH, Relling MV, Krynetski EY, Evans WE. Polymorphism of the thiopurine $S$-methyltransferase gene in African-Americans. Hum Mol Genet. 1999;8:371-376.

9. Ameyaw MM, Collie-Duguid ES, Powrie RH, Ofori-Adjei D, McLeod HL. Thiopurine methyltransferase alleles in British and Ghanaian populations. Hum Mol Genet. 1999;8:367-370.

10. Booth RA, Ansari MT, Loit E, et al. Assessment of thiopurine $S$ methyltransferase activity in patients prescribed thiopurines: a systematic review. Ann Intern Med. 2011;154:814-823. doi: 10.1059/0003-4819-154-12-201106210-00009.
11. Schaeffeler E, Fischer C, Brockmeier D, et al. Comprehensive analysis of thiopurine $S$-methyltransferase phenotype-genotype correlation in a large population of German-Caucasians and identification of novel TPMT variants. Pharmacogenetics. 2004; 14:407-417. doi:00008571-200407000-00004.

12. Gisbert JP, Gomollon F. Thiopurine-induced myelotoxicity in patients with inflammatory bowel disease: a review. Am J Gastroenterol. 2008;103:1783-1800. doi:10.1111/j.1572-0241.2008. 01848.x.

13. Kwan LY, Devlin SM, Mirocha JM, Papadakis KA. Thiopurine methyltransferase activity combined with 6-thioguanine metabolite levels predicts clinical response to thiopurines in patients with inflammatory bowel disease. Dig Liver Dis. 2008;40:425432. doi:10.1016/j.dld.2008.01.003.

14. Ansari A, Hassan C, Duley J, et al. Thiopurine methyltransferase activity and the use of azathioprine in inflammatory bowel disease. Aliment Pharmacol Ther. 2002;16:1743-1750.

15. Sparrow MP, Hande SA, Friedman S, et al. Allopurinol safely and effectively optimizes tioguanine metabolites in inflammatory bowel disease patients not responding to azathioprine and mercaptopurine. Aliment Pharmacol Ther. 2005;22:441-446. doi: 10.1111/j.1365-2036.2005.02583.x.

16. Sparrow MP, Hande SA, Friedman S, Cao D, Hanauer SB. Effect of allopurinol on clinical outcomes in inflammatory bowel disease nonresponders to azathioprine or 6-mercaptopurine. Clin Gastroenterol Hepatol. 2007;5:209-214. doi:10.1016/j.cgh.2006. 11.020.

17. Ansari A, Patel N, Sanderson J, O’Donohue J, Duley JA, Florin TH. Low-dose azathioprine or mercaptopurine in combination with allopurinol can bypass many adverse drug reactions in patients with inflammatory bowel disease. Aliment Pharmacol Ther. 2010;31:640-647. doi:10.1111/j.1365-2036.2009.04221.x.

18. Rundles RW. Effects of allopurinol on 6-mercaptopurine therapy in neoplastic diseases. Ann Rheum Dis. 1966;25:655-656.

19. Chocair P, Duley J, Simmonds HA, et al. Low-dose allopurinol plus azathioprine/cyclosporin/prednisolone, a novel immunosuppressive regimen. Lancet. 1993;342:83-84. doi:0140-6736(93) 91287-V.

20. Blaker PA, Arenas M, Fairbanks L, Irving P, Marinaki AM, Sanderson J. A biochemical mechanism for the role of allopurinol in TPMT inhibition. DDW. 2011;140:S769.

21. Duley JA, Chocair PR, Florin TH. Observations on the use of allopurinol in combination with azathioprine or mercaptopurine. Aliment Pharmacol Ther. 2005;22:1161-1162. doi:10.1111/ j.1365-2036.2005.02703.x.

22. Roberts RL, Gearry RB, Bland MV, et al. Trinucleotide repeat variants in the promoter of the thiopurine $S$-methyltransferase gene of patients exhibiting ultra-high enzyme activity. Pharmacogenet Genomics. 2008;18:434-438. doi:10.1097/FPC.0b013e 3282f85e4701213011-200805000-00007.

23. Lindqvist M, Haglund S, Almer S, et al. Identification of two novel sequence variants affecting thiopurine methyltransferase enzyme activity. Pharmacogenetics. 2004;14:261-265. doi:000 08571-200404000-00006.

24. Kham SK, Soh CK, Aw DC, Yeoh AE. TPMT*26 (208F $\rightarrow$ L), a novel mutation detected in a Chinese. Br J Clin Pharmacol. 2009;68:120-123. doi:10.1111/j.1365-2125.2009.03405.x.

25. Gonzalez-Lama Y, Bermejo F, Lopez-Sanroman A, et al. Thiopurine methyl-transferase activity and azathioprine metabolite concentrations do not predict clinical outcome in thiopurinetreated inflammatory bowel disease patients. Aliment Pharmacol Ther. 2011;34:544-554. doi:10.1111/j.1365-2036.2011.04756.x. 